\title{
Developing and evaluating threshold-based algorithms to detect drinking behavior in dairy cows using reticulorumen temperature
}

\author{
J. A. Vázquez-Diosdado, * G. G. Miguel-Pacheco, ${ }^{*}$ Bobbie Plant, Tania Dottorini, Martin Green, \\ and Jasmeet Kaler† \\ School of Veterinary Medicine and Science, University of Nottingham, Sutton Bonington Campus, Leicestershire LE12 5RD, United Kingdom
}

\section{ABSTRACT}

In this study, we assessed for the first time the use of a reticuloruminal temperature bolus and a thresholding method to detect drinking events and investigated different factors that can affect drinking behavior. First, we validated the detection of drinking events using 16 cows that received a reticuloruminal bolus. For this, we collected continuous drinking behavior data for $4 \mathrm{~d}$ using video recordings and ambient and water temperature for the same $4 \mathrm{~d}$. After all the data were synchronized, we performed 2 threshold algorithms: a generalfixed threshold and a cow-day specific threshold algorithm. In the general-fixed threshold, a positive test was considered if the temperature of any cow fell below a fixed threshold; in the cow-day specific threshold, a positive test was considered when the temperature of specific cows fell below the threshold value deviations around the mean temperature of the cow for that day. The former was evaluated using a threshold varying between 35.7 and $39.5^{\circ} \mathrm{C}$, and the latter using the formula $\mu-\frac{n}{10} \sigma$, where $\mu=$ mean of the temperature of each cow for one day, $n=1,2, \ldots, 20$, and $\sigma=$ standard deviation of the temperature of each cow on that day. The performance of the validation of detection using each of the threshold types was computed using different metrics, including overall accuracy, precision, recall (also known as sensitivity), $F$-score, positive predictive value, negative predictive value, false discovery rate, false omission rate, and Cohen's kappa statistic. The findings of the first study showed that the cow-day specific threshold of $n=10$ performed better (true positives $=466 ;$ false positives $=167$; false negatives $=$ 165 ; true negatives $=8,416$ ) than using a general-fixed threshold of $38.1^{\circ} \mathrm{C}$ (true positives $=449$; false positives

Received February 6, 2019.

Accepted June 28, 2019.

*These authors contributed equally to this work.

†Corresponding author: Jasmeet.Kaler@nottingham.ac.uk
$=181$; false negatives $=182 ;$ true negatives $=8,402$ ). With the information gained in this first study, we investigated the different factors associated with temperature drop characteristics per cow: number of drops, mean amplitude of the drop, and mean recovery time. For this, we used data from 54 cows collected for almost $1 \mathrm{yr}$ to build a mixed-effect multilevel model that included days in milk, parity, average monthly milk production, and ambient temperature as explanatory variables. Cow characteristics and ambient temperature had significant effects on drinking events. Our results provide a platform for automated monitoring of drinking behavior, which has potential value in prediction of health and welfare in dairy cattle.

Key words: reticuloruminal temperature, drinking behavior, algorithm, dairy cattle, precision livestock

\section{INTRODUCTION}

In recent years, the development of precision livestock farming technology has facilitated not only the collection of real-time data but also the integration of this information within the overall monitoring of individual animals (Berckmans, 2014; Walton et al., 2018). Among these technologies is the collection of reticuloruminal temperature data through the use of reticular boluses that transmit data to a central computer using an active radiofrequency transmitter (Costa et al., 2016; Kovács et al., 2017; Lees et al., 2018). This allows farmers to continuously monitor cows' temperature, providing an alert when temperature goes out of a preset range; for example, during heat stress (Koltes et al., 2018). This technology could provide a high-quality, time-efficient approach to monitor the health of individual animals, thereby improving farmers' and animals' welfare.

Reticuloruminal temperature has been assessed as a potential prediction tool for estrus (Cooper-Prado et al., 2011), calving (Costa et al., 2016), dystocia (Kovács et al., 2017), and health status (Timsit et al., 2011) in cattle. In addition, reticuloruminal temperature can be affected by external variables such as water intake (Bewley et al., 2008b). This information could be used 
as a potential proxy to measure automatically drinking behavior in cattle.

Monitoring drinking behavior is important for milk production, health, and physiological status in dairy cows. Cows require between 24 and $136 \mathrm{~L}$ of water per day based on their lactation period (Murphy, 1992), drink on average $7.3( \pm 2.8)$ times per day, and prefer to drink between 0600 and $1900 \mathrm{~h}$, particularly after feeding and milking (Cardot et al., 2008). Factors affecting water intake have a direct effect on milk production; a reduction in water intake can decrease milk yield up to $26 \%$ (Steiger Burgos et al., 2001), whereas free access to water can increase milk production by up to $1.7 \mathrm{~L} / \mathrm{d}$ (Daros et al., 2019). It has been observed that cows increase their drinking bouts as temperature-humidity index (THI) increases; however, drinking bouts decrease once the THI is $>82$ (González Pereyra et al., 2010). In the case of health status, monitoring drinking behavior can be used to assess the development of health conditions such as those seen in calves during diarrhea events (Wenge et al., 2014) or to predict diseases or physiological states. Lukas et al. (2008) identified a negative association between water intake and calving and with fever and other diseases, whereas De Mol et al. (2001) was able to detect mastitis and other diseases using water intake events with a specificity of $86 \%$.

Previous monitoring studies have associated water intake with events when temperature is below $37.8^{\circ} \mathrm{C}$ or below 3 times the standard deviation of a cow temperature (Bewley et al., 2008a). Bewley et al. (2008b) observed that water intake and water temperature affected the size of the reticuloruminal temperature drop and the duration of the effect; the colder the water, the greater the temperature drop and longer the time to recover. Cold water at $5.1^{\circ} \mathrm{C}$ decreased reticuloruminal temperature by an estimated $9.2^{\circ} \mathrm{C}$, taking up to $3.5 \mathrm{~h}$ to return to the baseline temperature, whereas water at $34.3^{\circ} \mathrm{C}$ decreased reticuloruminal temperature by $2.2^{\circ} \mathrm{C}$, taking up to $2 \mathrm{~h}$ to return to baseline (Bewley et al., 2008b). However, the latter study did not control for other cow or environmental factors, and cows were observed under experimental conditions (e.g., feed and water access restricted for $2 \mathrm{~h}$ before and $3 \mathrm{~h}$ after study).

Reticuloruminal temperature can also vary depending on the cow breed, milk production level, and DIM (Bewley et al., 2008a; Liang et al., 2013; Stone et al., 2017). It has been observed that reticuloruminal temperature decreases as DIM and milk production increase (Bewley et al., 2008a; Stone et al., 2017); however, this latter association remains unclear. Although it could be expected that higher milk production will contribute to greater production of body heat, thus increasing reticuloruminal temperature, another study observed a negative association between milk production and reticuloruminal temperature (Liang et al., 2013).

The present investigation comprised 2 studies: the objective of the first was to validate the use of threshold algorithms to detect and determine drinking behaviors using a reticuloruminal temperature bolus. The objective of the second was to use the threshold algorithm from the first study to explore characteristics of drinking-related reticuloruminal temperature drops, including the effect of predictor variables such as ambient temperature, production, DIM, parity, and hour of the day. This work provides, for the first time, a comprehensive evaluation of the effect of drinking events on the reticuloruminal temperature of cows under field conditions.

\section{MATERIALS AND METHODS}

The present study was reviewed and approved by the Ethical Committee at the School of Veterinary Medicine and Science, University of Nottingham (Approval Number: 1895- 161109).

\section{Farm Management}

All data used in this paper were collected on a commercial dairy farm located in Worcestershire, United Kingdom. The herd comprised 432 Holstein Friesian milking cows with an annual yield of approximately $10,000 \mathrm{~kg} /$ cow. The barn had deep sand-bedded stalls and fans located at each end that were operational over the summer months. Cows had ad libitum access to fresh water from 3 water troughs located around the barn and to a TMR pushed up 3 times a day after milking in one lane, to which cows had access from both sides. Cows were moved to pasture for approximately 3 $\mathrm{h}$ after the morning milking year round, weather permitting. While grazing, cows had ad libitum access to water.

\section{Reticuloruminal Boluses and Ambient Temperature}

For both studies (1 and 2), boluses (SmaXtec Animal Care GmbH, Graz, Austria) were used to collect reticuloruminal temperature data; boluses were administered by farm personnel using an oral applicator. Each bolus was powered by an internal lithium metal battery with a life of up to $4 \mathrm{yr}$. The internal antenna enabled communication with an external receiver (SmaXtec Animal Care Technology). According to the product specification, temperatures between 0 and $80^{\circ} \mathrm{C}$ could be measured with an accuracy of $\pm 0.05^{\circ} \mathrm{C}$ (SmaXtec, 2018). The bolus has been validated for use in dairy cattle (Ammer et al., 2016a) and has been used to accurately 
Table 1. Drinking behavior definitions used in study 1 (validation of detection drinking events) based on Cardot et al. (2008)

\begin{tabular}{ll}
\hline Drinking behavior & Description \\
\hline Drinking sip & A cow introduces its nose onto the water and finishes when she raises her head above water level \\
Drinking bout & Aggregation of one of several drinking events of water sipping
\end{tabular}

measure dairy cattle reticuloruminal temperature under different experimental settings (Gasteiner et al., 2009; Ammer et al., 2016b). The bolus also contained an accelerometer generating an activity index between 1 and 100. Activity and reticuloruminal temperature were recorded every $10 \mathrm{~min}$ and uploaded wirelessly to one of the several receivers within the barns when the cow came into range. Data were then logged on an Excel spreadsheet (Microsoft Corp., Redmond, WA), including the date and time when activity and temperature were recorded. For the present study, activity data were not used. Ambient temperature was collected every 10 min using one ambient temperature sensor located 2.5 $\mathrm{m}$ above ground level in the middle of the barn (13.71 $\mathrm{m}$ length $\times 12.19 \mathrm{~m}$ width barn).

\section{Study 1: Validation of Detection of Drinking Events}

Drinking Behavior. The validation study was designed to assess the detection of drinking events by detecting temperature changes using a threshold method. The drinking behavior of 16 cows with reticular boluses was recorded continuously for $4 \mathrm{~d}$ using CCTV cameras (5 Mp, 30 m IR; Hikvision Digital Technology Co. Ltd., City of Industry, CA) that were mounted $2 \mathrm{~m}$ above each of the 3 water troughs. The cameras were set to record at high quality (video format HEVC, H.265; and at 2,944 $\times 1,656$ pixels quality) and at 20 frames/s. Cameras were connected to a $4-\mathrm{MB}$ video recorder (Hikvision Digital Technology Co. Ltd.) from where video data were downloaded once the 4-d observation period was completed. Water and ambient temperature were also collected; water temperature was monitored using the same reticular boluses immersed in each water trough for the duration of the study. Ambient temperature was recorded using the previously described sensor.

An experienced behavioral researcher (G. G. M.-P.) scored the video recordings retrospectively to determine the times when cows were drinking, including the time spent on this behavior. To ease identification on the video recordings, each cow was spray marked on the back with a number from 1 to 20 ; when number identification was not possible, cows were identified on the videos by their coat patterns.

Videos were analyzed using drinking behavior definitions as described by Cardot et al. (2008) and given in Table 1. Accordingly, each drinking behavior bout started with the first sip of water and finished when at least 4 min elapsed without any drinking. A drinking sip started when the cow introduced its nose onto the water and finished when she raised her head above water level. A drinking bout could be made up of one or several events of water sipping. Two variables were obtained from the video analysis: number of drinking bouts and duration of drinking bouts.

Data Processing and Analysis. Drinking behavior variables (number and duration of drinking bouts) were synchronized with reticular, ambient, and water trough temperatures. Synchronization was carried out in 2 steps. First, reticular, ambient, and water trough temperatures were synchronized by matching timestamps. In the second step, behavioral information (number and duration of drinking bouts) was combined with sensor temperature information. For this second step, binary labels for drinking behaviors (drinking/not drinking) were assigned, within the 10-min resolution of the reticular temperature boluses, whenever there was or was not a drinking bout.

Validation of the Detection of Drinking Events. The detection of drinking events was validated using a reticuloruminal temperature threshold method using 2 threshold algorithms: a general-fixed threshold and a cow-day specific threshold. We used the threshold method because of its simplicity and effectiveness in detecting temperature dropping events that occurred as a result of drinking events, in a time series (Tong, 2011). Moreover, simple threshold methods have shown to be accurate in classifying different activities in cows (Vázquez Diosdado et al., 2015) when using accelerometer data. The algorithms were as follows:

(1) General-fixed threshold algorithm: A single temperature threshold was used for all cows to define a temperature drop (i.e., if the temperature of any cow fell below the general threshold, then it was considered a positive test). The general-fixed threshold type was evaluated using a threshold varying between 35.7 and $39.5^{\circ} \mathrm{C}$ in increments of $0.2^{\circ} \mathrm{C}$.

(2) Cow-day specific threshold algorithm: This threshold was computed using the formula $\mu-\frac{n}{10} \sigma$, where $n=1,2, \ldots, 20(n$ is a parameter 
that describes the different decreasing values of the cow-day specific threshold algorithm), $\mu$ is the mean of the temperature of each cow for one day, and $\sigma$ is the standard deviation of the temperature of each cow on that day. The cow-day specific formula looks at deviations below the mean core temperature of the cow using both the mean and standard deviation of the cow temperature on the specific day. Therefore, a large $n$ (e.g., $n=20$ ) represents a low temperature threshold $(\mu-2 \sigma)$, whereas a small $n$ (e.g., $n=$ 1) represents a high temperature threshold $\left(\mu-\frac{1}{10} \sigma\right)$.

Performance of the Validation. Algorithm performance was evaluated using different metrics which included overall accuracy, precision, recall (also known as sensitivity), $F$-score, positive predictive value (PPV), negative predictive value (NPV), false discovery rate (FDR), and false omission rate (FOR), as defined in Dohoo et al. (2009), and Cohen's kappa ( $\boldsymbol{\kappa}$ ) statistic (Ben-David, 2008):

$$
\text { Cohen's } \kappa=\frac{p_{o}-p_{e}}{1-p_{e}},
$$

where $p_{o}$ is the relative observed agreement between the observed drinking event and the prediction of the drinking event, and $p_{e}$ is the hypothetical probability of change agreement. We also computed additional measures of the number of true positives (TP) divided by the number of drinking events, the number of false positives (FP) divided by the number of drinking events, the number of false negatives (FN) divided by the number of drinking events, and the number of predicted positives $(\mathrm{TP}+\mathrm{FP})$ divided by the number of drinking events. Receiver operating characteristic (ROC) curves, using both the general and the cowspecific thresholds, were obtained by computing the true positive rate (TPR) and the false positive rate $(\mathrm{FPR})$, where $\mathrm{TPR}=\mathrm{TP} /(\mathrm{TP}+\mathrm{FN})$ and $\mathrm{FPR}=\mathrm{FP} /$ $(\mathrm{FP}+\mathrm{TN})$ and $\mathrm{TN}=$ true negative.

\section{Study2: Factors Associated with Drinking Event Characteristics}

Data Processing. Seventy-six cows were selected for this study. Reticular boluses were placed into cows at different times between June 2016 and August 2017 by farm personnel using an oral applicator and provided raw temperature data points every $10 \mathrm{~min}$. Monthly milk production, DIM, and parity data were collected using the on-farm management software UNIFORMAgri (Assen, the Netherlands). Cows produced on average $41.82( \pm 10.45) \mathrm{L}$ of milk per day and had an average of $140.62( \pm 101.68)$ DIM and a parity range between 1 and 7 . Cows in the data set that were diagnosed with either mastitis or another disease (e.g., retained fetal membranes) were removed entirely from the data set. This reduced the data set to 54 individual cows, with data collected from June 10, 2016, to April 12, 2018. From this group of 54 individual cows, we removed data from $3 \mathrm{wk}$ before and $3 \mathrm{wk}$ after a treatment event was reported (e.g., treated cows). Days where there was no information on DIM, production, or parity were also removed from the data set, giving a total of $3,474,983$ raw temperature data points. Therefore, data used for the analysis contained only information at times when cows were believed to be healthy, hence removing effects of illness. Additionally, temperature outliers (values $<29^{\circ} \mathrm{C}$; Bewley et al., 2008b), representing $0.0705 \%$ of the raw filtered data for the 54 cows, were removed from the data set.

Mixed-Effect Modeling. The potential associations between drinking event characteristics and explanatory variables were investigated using mixed-effect models. Three mixed-effect models were fitted, 1 for each of the 3 different drinking event outcomes: (1) the number of drinking events per cow per day, (2) the mean amplitude of cow drinking events per day, and (3) the mean recovery time of the drinking event per cow day. The number of drinking events was determined using the cow-day specific threshold method from study 1 , where an event was detected when the temperature was below a specific threshold. The amplitude of a drinking event was computed as the difference between the minimum temperature within a drinking event and the temperature at the first point it fell below the threshold. Recovery time was computed as the duration from the point at which temperature went below the threshold until it returned to the threshold value. The statistical models can be summarized as follows:

$$
\begin{aligned}
Z_{i j}= & \widehat{\beta_{0 i j}}+\widehat{\beta_{1 i j}} X_{1 i j}+\widehat{\beta_{2 i j}} X_{2 i j}+\widehat{\beta_{3 i j}} X_{3 i j} \\
& +\widehat{\beta_{4 i j}} X_{4 i j}+\widehat{\beta_{5 i j}} X_{5 i j}+\widehat{V_{o j}}+\widehat{\varepsilon_{i j}},
\end{aligned}
$$

where $Z$ represents a transformed version of the drop characteristic, $i$ represents a repeated measurement of $Z, j$ represents a cow, and $\beta_{0}$ represents the intercept; $\widehat{V_{o j}}$ is a random effect for cow, and $\varepsilon_{i j}$ is the residual error in the transformed space. Thus, $\widehat{\beta_{1 i j}}, \widehat{\beta_{2 i j}}, \widehat{\beta_{3 i j}}, \widehat{\beta_{4 i j}}$, and $\widehat{\beta_{5 i j}}$ represent vector coefficients for explanatory variables $\left(X_{1}\right.$ to $\left.X_{5}\right)$. In each model, the $Z$ drop characteristic was transformed using 
a Box-Cox power transformation (Box and Cox, 1964; Sakia, 1992) defined according to the formula

$$
Z=\left\{\begin{array}{cc}
y^{\lambda}-1 / \lambda & \lambda \neq 0 \\
\log (y) & \lambda=0
\end{array}\right.
$$

where $y$ was the variable to be transformed and $\lambda$ was the transformation parameter. In each of the mixed models, the transformation parameter $\lambda$ was obtained using the maximum likelihood (Seaks and Layson, 1983). Box-Cox power transformation was performed using the Box-Cox function within the MASS package (Venables and Ripley, 2002) in R (R Core Team, 2017).

All models were set at the daily level resolution, meaning that the number of temperature drops was obtained for each cow each day; for the amplitude and recovery time, the mean of each day was used. The explanatory variables ambient temperature (at the time of the drop), production, parity, DIM, and hour of the day were tested in each model. Days in milk, parity, and hour were treated as categorical variables; DIM was split into 3 categories-DIM phase 1, 2, and 3 corresponding to ranges $1-75,76-200$, and > 200 DIM, respectively. The distribution of predictor variables (DIM and parity) is shown in Table 2. Because of the percentage of data points in parities 5,6 , and 7 , these categories were grouped into a single category labeled parity 5 and above. Hour of the day was grouped into 3 categories: group 1 , between 0000 and $0800 \mathrm{~h}$, group 2, between 0801 and $1600 \mathrm{~h}$, and group 3, between 1601 and $2400 \mathrm{~h}$. These categories were selected based on milking patterns, because drinking behavior occurs more frequently after milking (Cardot et al., 2008).

Computation of the number of drops, amplitude of the drop, and recovery time for all models was performed using custom-made scripts written in Matlab and Statistics Toolbox Release (2017a). The mixed model was fitted using the "Imer" function (Bates et al., 2015) from the lme4 package in $\mathrm{R}$ (R Core Team, 2017). Inference for the parameters of the mixed model in the original scale was obtained using the procedure developed by Maruo et al. (2017), who proposed a method to obtain the model median difference between any 2 groups on the original scale. This model median is readily interpretable because it is an estimator on the original scale. According to this procedure, the model median difference between groups $g_{1}$ and $g_{2}$ is given by $\delta_{\left(g_{1} ; g_{2}\right)}=\xi_{g_{1}}-\xi_{g_{2}}$, where $\xi_{g}=\left(\lambda \eta_{g}+1\right)^{1 / \lambda}, \eta_{g}$ represents the model mean on the transformed scale, and $\xi_{g}$ is the model median in the original scale.

For DIM, median differences between DIM1 and DIM2, DIM1 and DIM3 were computed. For parity, median differences between categories parity 1 and parity 2 , parity 1 and parity 3 , parity 1 and parity 4 , and parity 1 and parity 5 were computed. For hour, median difference between categories hour 1 and hour 2 , and hour 1 and hour 3 were computed. For ambient temperature, we created 2 categories: Temp_15_16 (temperature between $15^{\circ} \mathrm{C}$ and $16^{\circ} \mathrm{C}$ ) and Temp_16_17 (temperature between $16^{\circ} \mathrm{C}$ and $17^{\circ} \mathrm{C}$ ), to measure the effect that a change in $1^{\circ} \mathrm{C}$ in ambient temperature will have on the different drop characteristics in the original scale. Ambient temperature was used here as a proxy of water temperature because we expected them to be highly correlated (water on the tanks was not heated). For production, we created 2 categories Prod_41_42 (production between 41 and $42 \mathrm{~L}$ ) and Prod_42_43 (production between 42 and $43 \mathrm{~L}$ ), to measure the effect that a change of $1 \mathrm{~L}$ more milk in production would have on the temperature drop characteristics in the original scale. All of these categories were defined to model differences between categories at the original scale following the procedure developed in Maruo et al. (2017). All statistical analysis were completed using the $\mathrm{R}$ language and software (R Core Team, 2017).

Sensitivity Analysis. To determine how sensitive the model parameter estimates were to the definition of a reticular temperature drop, individual models were rebuilt and parameter estimates re-evaluated using the cow-day specific threshold for values of $n=10,12$, and 15. This procedure was performed using an individual mixed model, as described above for each of the drinking event characteristics.

\section{RESULTS}

\section{Study 1: Validation of Detection Drinking Events}

In Figure 1, we show an example of the reticuloruminal temperature of the cow and "ground truth" (defined as the instances where a drinking event was observed)

Table 2. Number of data points (measured in days) within each DIM and parity category ${ }^{1}$

\begin{tabular}{lcc}
\hline Predictor & $\begin{array}{c}\text { No. of data } \\
\text { points }\end{array}$ & $\begin{array}{c}\text { Percentage } \\
\text { of total }\end{array}$ \\
\hline DIM phase 1 & 3,954 & 36.45 \\
DIM phase 2 & 2,665 & 24.57 \\
DIM phase 3 & 4,227 & 38.97 \\
Parity 1 & 1,263 & 11.64 \\
Parity 2 & 1,904 & 17.55 \\
Parity 3 & 4,303 & 39.67 \\
Parity 4 & 2,299 & 21.19 \\
Parity 5 & 546 & 5.03 \\
Parity 6 & 328 & 3.02 \\
Parity 7 & 203 & 1.87 \\
\hline
\end{tabular}

${ }^{1}$ DIM was split into 3 categories: DIM phase 1,2 , and 3 , which corresponded to ranges $1-75,76-200$, and >200 DIM. 


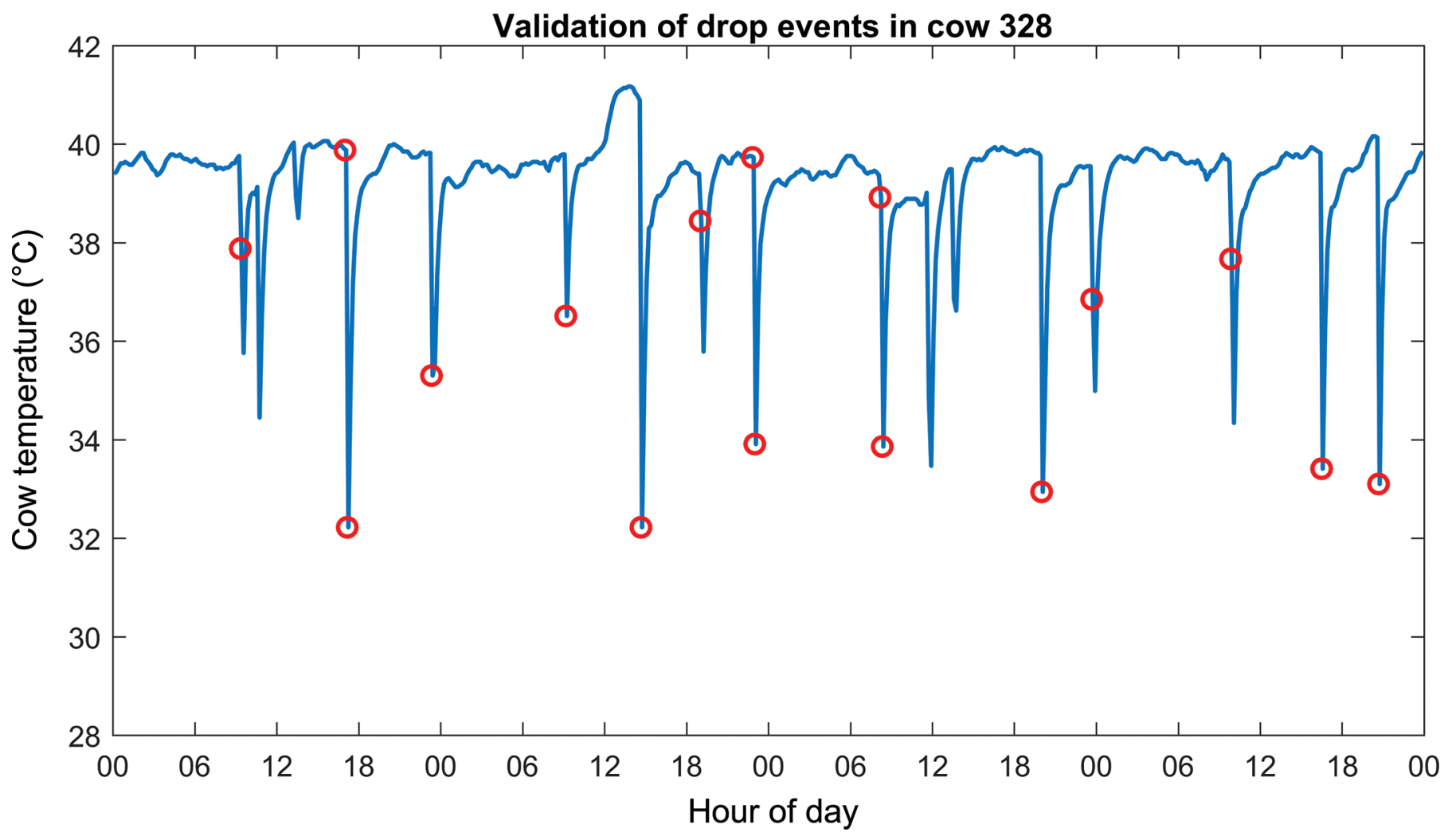

Figure 1. Example of reticuloruminal temperature changes in one cow (no. 328) over 4 d, illustrating temperature drops and ground truth behavior (red circles).

drinking events for the $4 \mathrm{~d}$ of the validation study. We recorded a total of 631 ground truth drinking events for the duration of the study.

Performance of Drinking Event Detection: General-Fixed Threshold Algorithm. The performance of the algorithm for detection of drinking events using the general-fixed threshold is shown in Figure 2. Both accuracy and specificity reached a plateau for threshold values between $35.7^{\circ} \mathrm{C}$ and $38.3^{\circ} \mathrm{C}$, at which point they decreased rapidly. Recall increased with an increased threshold value to a maximum of $87.5 \%$ for a threshold of $39.5^{\circ} \mathrm{C}$. Precision decreased with an increased threshold, and the reduction in precision was particularly steep above $37.9^{\circ} \mathrm{C}$. The $F$-score and Cohen's $\kappa$ increased from $35.3^{\circ} \mathrm{C}$ to $37.5^{\circ} \mathrm{C}$ and decreased for values above $37.5^{\circ} \mathrm{C}$; NPV and $\mathrm{FOR}$ remained almost constant for all the threshold values. Positive predictive value decreased with an increasing threshold, and the rate of decrease of PPV was higher for threshold values above $37.7^{\circ} \mathrm{C}$. False discovery rate increased with increasing threshold values and increased markedly above a threshold of $37.7^{\circ} \mathrm{C}$. Therefore, the best range for maximizing the sensitivity (recall) of the detection of drinking events while maintaining a high level of precision (PPV) and specificity appeared to be located between $37.1^{\circ} \mathrm{C}$ and $38.1^{\circ} \mathrm{C}$. In this range, the FDR was below $28.73 \%$. The total number of TP, FP, total predicted positives, $\mathrm{FN}$, and $\mathrm{TN}$ for this range $\left(37.1^{\circ} \mathrm{C}-38.1^{\circ} \mathrm{C}\right)$ is shown in Table 2 .

A threshold of $38.1^{\circ} \mathrm{C}$ produced a total of 630 positives (99.84\% of the number of observed events) which was the closest to the 631 ground truth drinking events recorded. Using this threshold, 181 false positives (28.68\% of the total number of events) and 182 false negatives were computed ( $28.84 \%$ of the total number of events).

Performance of the Drinking Event Detection: Cow-Day Specific Threshold Algorithm. The performance of the detection of drinking events using the cow-day threshold and the different performance metrics are shown in Figure 3. Both accuracy and specificity reached a plateau for threshold values between $n=20$ and $n=1$, at which point they decreased rapidly. Recall increased with an increased threshold value, with a maximum of $85.26 \%$ for a threshold of $n=1$. Precision decreased with an increasing threshold and decreased particularly quickly for threshold values above $n=10$. The $F$-score and Cohen's $\kappa$ reached a plateau from $n$ $=20$ to $n=10$ and decreased for values above $n=10$. Negative predictive value and FOR remained almost 

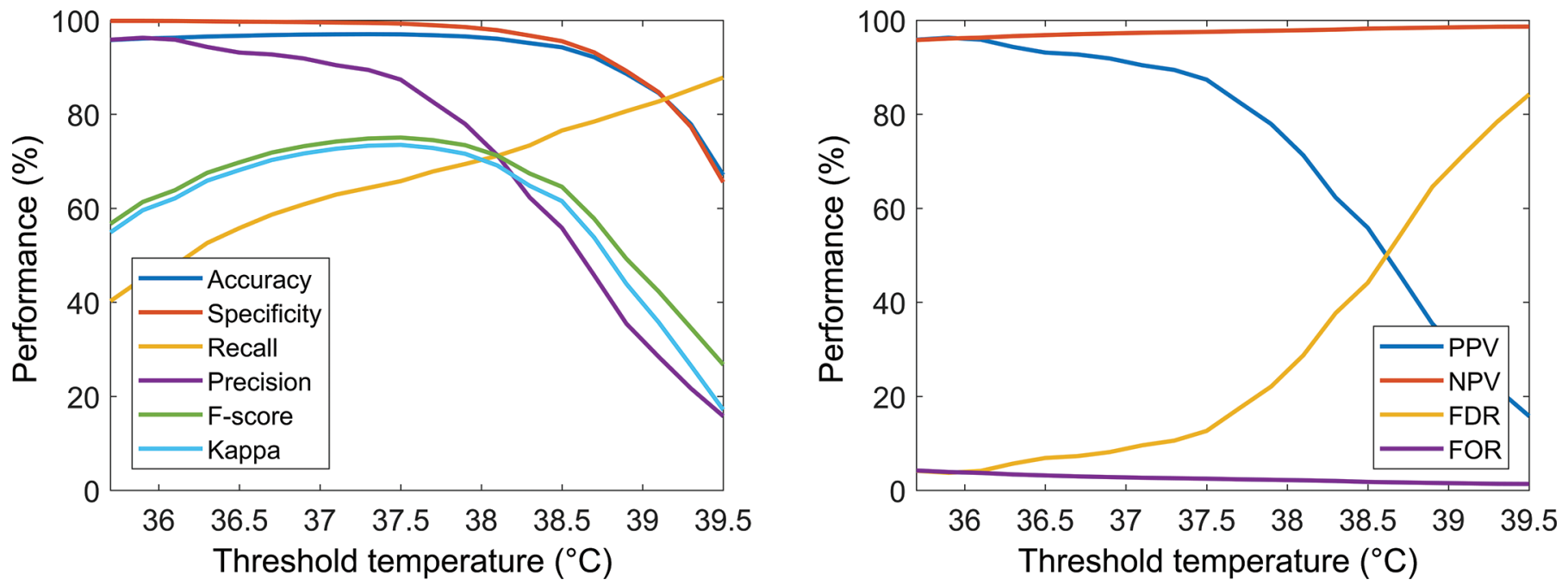

Figure 2. Performance of validation using accuracy, specificity, recall, precision, $F$-score, Cohen's kappa, positive predictive value (PPV), negative predictive value (NPV), false discovery rate (FDR), and false omission rate (FOR) for the general fixed threshold algorithm varying in a temperature threshold range between 35.7 and $39.5^{\circ} \mathrm{C}$.

constant for all the threshold values. Positive predictive power decreased with an increasing threshold, and the rate of decrease was higher for threshold values above $n=10$. False discovery rate increased with increasing threshold values and the rate was higher for values above $n=10$. Therefore, the best range for maximizing the sensitivity (recall) of the detection of drinking events while maintaining a high level of precision (PPV) and specificity was between $n=15$ and $n=10$. In this range, FDR was below $13.68 \%$. The total number of
TP, FP, total predicted positives, FN, and TN for the range $n=15-10$ are shown in Table 3 .

A threshold of $n=10$ produced a total of 633 positives (100.32\% of the total number of observed events), which was the closest to the 631 ground drinking events recorded. Using this threshold, $167 \mathrm{FP}$ ( $26.47 \%$ of the total number of events) and $162 \mathrm{FN}$ (26.15\% of the total number of events) were computed.

The exact performance values using the 2 threshold types can be seen in the supplementary material
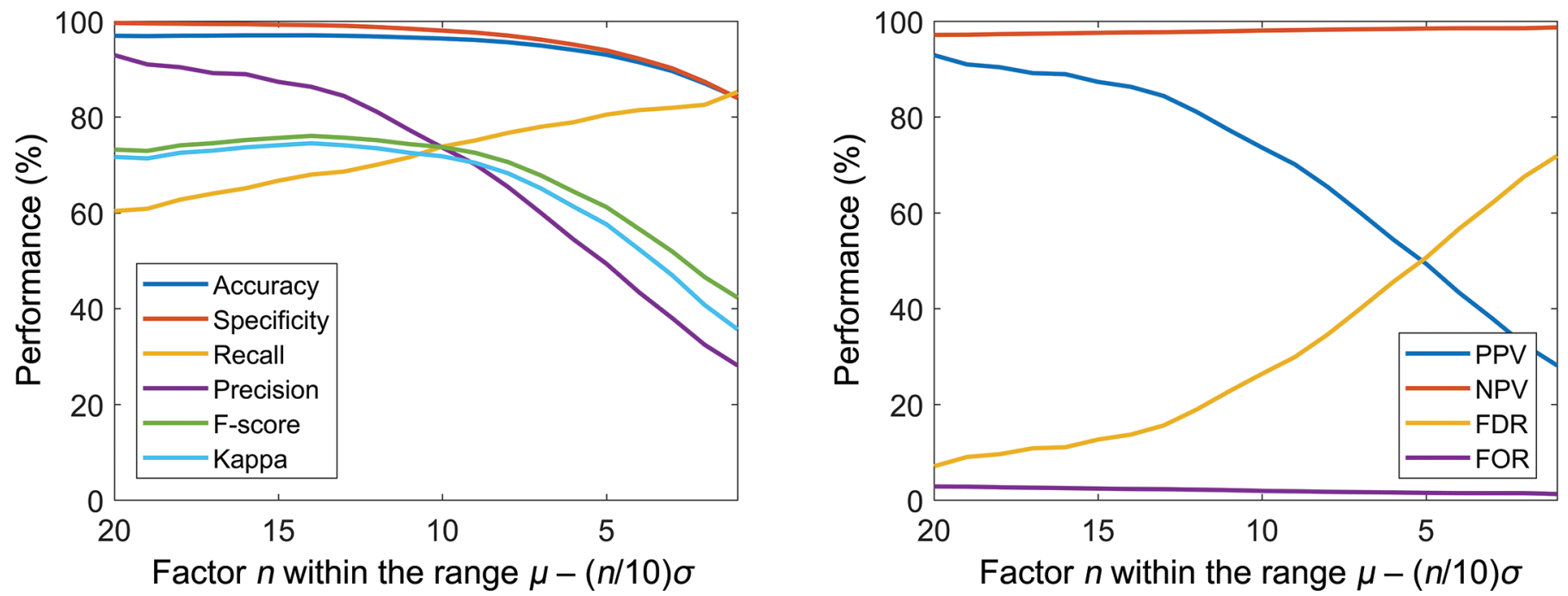

Figure 3. Performance of validation using accuracy, specificity, recall, precision, F-score, Cohen's kappa, positive predictive value (PPV), negative predictive value (NPV), false discovery rate (FDR), and false omission rate (FOR) for the cow-day specific threshold that varied according to the formula $\mu-\frac{n}{10} \sigma$, where $n=1,2, \ldots, 20, \mu=$ mean of the temperature of each cow for one day, $n=1,2, \ldots, 20$, and $\sigma=$ standard deviation of the temperature of each cow on that day. 


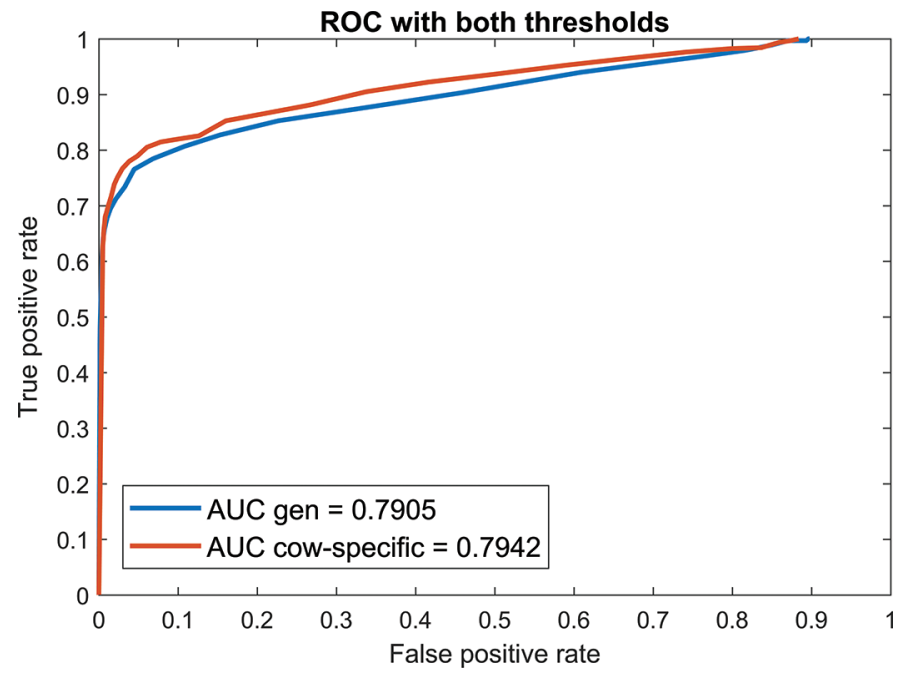

Figure 4. Performance of validation using receiver operating characteristic (ROC) curves using both the general (gen) and cow-specific thresholds. The plot shows the area under the curve (AUC) for both thresholds.

(Supplemental Tables S1 and S2; https://doi.org/10 .3168/jds.2019-16442). We also provide information on the TP, FP, total predicted positives, FN, and TN over the whole range of the 2 thresholds. A comparison of the ROC curves using both the general and the cowspecific thresholds is shown in Figure 4. Additionally, we computed the area under the curve (AUC) for each of the thresholds, which showed a slightly higher AUC (0.7942) for the cow-day specific threshold compared with that $(0.7905)$ for the general threshold.

\section{Study 2: Factors Associated with Drinking Event Characteristics}

The sensitivity analysis was performed using the cow-day specific threshold algorithm as this produced the best performance result. Results of the sensitivity analysis conducted using multilevel mixed models for each of the different drinking event characteristics with values $n=10,12$, and 15 are shown in Table 4 .

Number of Drinking Events. The median number of drinking events (intercept; Table 4) varied between 5.84 and 6.09 for the different threshold values $(n=$ 15,12 , and 10). The number of drinking events was significantly higher at ambient temperatures in the 16 to $17^{\circ} \mathrm{C}$ range compared with the 15 to $16^{\circ} \mathrm{C}$ temperature range. Cows in DIM 2 and DIM 3 had significantly fewer drinking events compared with cows in DIM 1, and cows in DIM 3 had fewer drinking events than cows in DIM 2. Cows in parity 2 and parity 3 had significantly fewer drinking events compared with cows in parity 1 .

Amplitude of the Drinking Event. The amplitude of the drinking event varied between $2.37^{\circ} \mathrm{C}$ and $2.82^{\circ} \mathrm{C}$ for the different threshold values $(n=15,12$, and 10$)$. The amplitude of the drinking event was significantly smaller at ambient temperatures in the $16-17^{\circ} \mathrm{C}$ range compared with temperatures in the $15-16^{\circ} \mathrm{C}$ range. Cows in DIM 2 and DIM 3 had significantly larger amplitude of drinking event compared with cows in DIM 1 , and amplitude of the drinking event for cows in DIM 3 was larger than that of cows in DIM 2. Cows in parity 2 , parity 3 had a significantly larger amplitude of the drinking event compared with cows in parity 1 . Cows in parity 5 had a significantly smaller amplitude of the drinking event compared with cows in parity 1 .

Recovery Time. The recovery time of the drinking event varied between 29.98 and 35.55 min for the different threshold values $(n=15,12$, and 10). Cows in DIM 3 had a significantly higher recovery time compared with cows in DIM 1. Cows in parity 4 had a significant lower recovery time of the drinking event compared with cows in parity 1 .

In summary, we identified a significant effect from DIM 2 and DIM 3 and parity 2 and parity 3 in the number of drinking events for all 3 threshold values. Additionally, we identified a significant effect from ambient temperature, DIM 2 and DIM 3, and parity 2

Table 3. Number of true positives (TP), false positives (FP), false negatives (FN), number of predicted positives $(\mathrm{TP}+\mathrm{FP})$, and true negatives $(\mathrm{TN})$ using the cow-day specific threshold that varied according to the formula $\mu-\frac{n}{10} \sigma$, with $n=15$ to $10^{1}$

\begin{tabular}{lccccc}
\hline $\begin{array}{l}\text { Threshold } \\
\text { value } n\end{array}$ & TP & FP & TP+FP & FN & TN \\
\hline 15 & 421 & 61 & 482 & 210 & 8,522 \\
14 & 429 & 68 & 497 & 202 & 8,515 \\
13 & 433 & 80 & 513 & 198 & 8,503 \\
12 & 442 & 103 & 545 & 189 & 8,480 \\
11 & 452 & 133 & 585 & 179 & 8,450 \\
10 & 466 & 167 & 633 & 165 & 8,416 \\
\hline
\end{tabular}

${ }^{1} \mu=$ mean of the temperature of each cow for one day, $n=1,2, \ldots, 20$, and $\sigma=$ standard deviation of the temperature of each cow on that day 


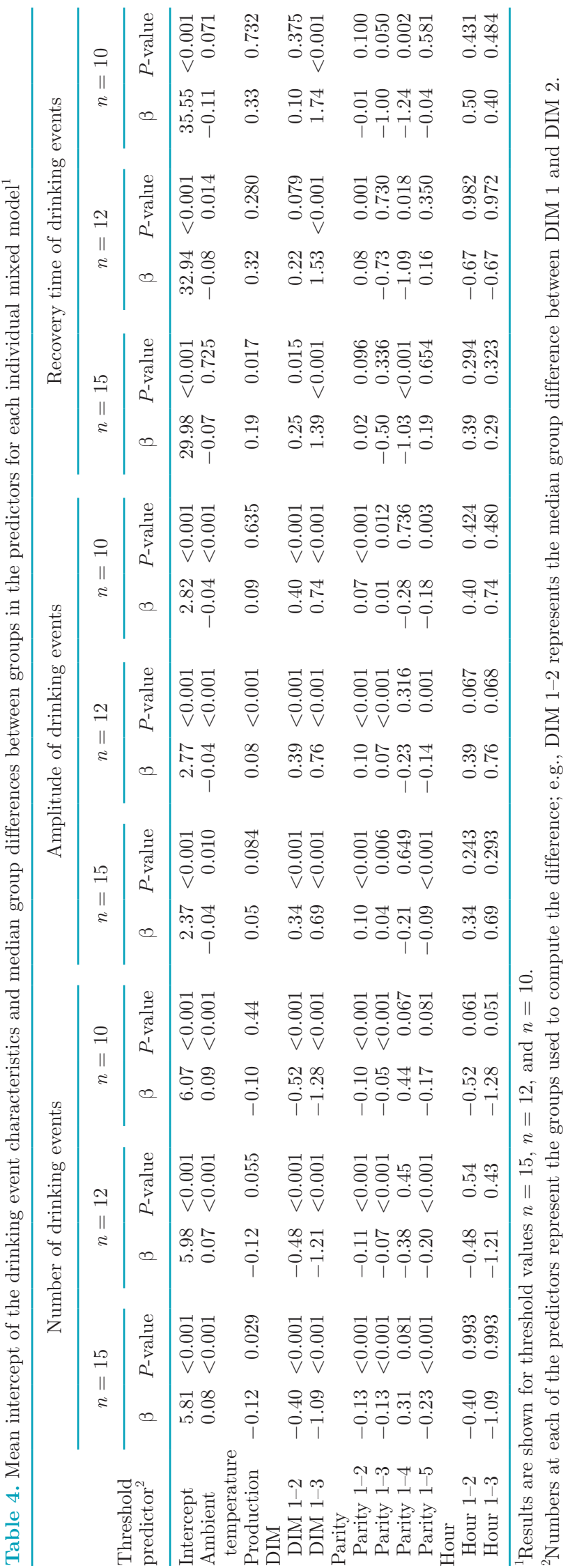

and parity 5 using the 3 different threshold values. For the recovery time of the drinking event, we identified a significant effect from DIM 3 using the 3 threshold values.

\section{DISCUSSION}

To our knowledge, this is the first study to develop and compare algorithms using reticuloruminal bolus temperature to detect drinking behavior in dairy cows and study what factors could affect drinking events as detected by temperature. We were able to robustly validate the use of a simple threshold algorithm to detect drinking events through temperature drops in a short trial $(4 \mathrm{~d})$ and to use this method to investigate associations between drinking events and cow characteristics on a larger longitudinal data set. Validation of threshold algorithms for detection of drinking events was performed using 2 types of thresholds (generalfixed and cow-day specific) and over a wide range of values that provided a more robust assessment than using a single threshold value, as seen in a previous study (Boehmer et al., 2009). This was one of the key strengths of this study because we were able to evaluate different performance parameters and trade-offs, as discussed below.

The general-fixed threshold algorithm showed that the best threshold values were located between $37.1^{\circ} \mathrm{C}$ and $38.1^{\circ} \mathrm{C}$. Within this range, when maximizing recall (high level of specificity and accuracy and relatively high level of precision), the best value was $38.1^{\circ} \mathrm{C}$. In the case of the cow-day specific threshold algorithm, we observed that the best result was located between $n=15$ and $n=10$; and when maximizing recall (sensitivity) and maintaining a high level of specificity and accuracy and relatively high level of precision, the best value was located at $n=10$. Overall, the cow-day specific threshold of $n=10$ performed slightly better $(\mathrm{TP}=466 ; \mathrm{FP}=167 ; \mathrm{FN}=165 ; \mathrm{TN}=8,416)$ than using a $38.1^{\circ} \mathrm{C}$ general-fixed threshold $(\mathrm{TP}=449 ; \mathrm{FP}$ $=181 ; \mathrm{FN}=182 ; \mathrm{TN}=8,402 ;$ Tables 2 and 3 ). This is possible because the cow-day specific threshold analysis included individual cow reticulorumen temperature, which may be affected by external factors such as water and ambient temperature, humidity, and seasonality. In contrast, the general-fix threshold did not incorporate any of these factors.

Previous studies have suggested that drinking events can be detected using a fixed threshold value of $37.7^{\circ} \mathrm{C}$ (Cooper-Prado et al., 2011; Costa et al., 2016). This was based on previous studies that used a small sample ( $\mathrm{n}=9$ cows; Boehmer et al., 2009), or few water intake observations per cow (e.g., 3 data points per cow; Bewley et al., 2008b; Boehmer et al., 2009), or col- 
lected data under controlled experimental conditions (e.g., oral drench, water and feed restricted for up to 21 h; Bewley et al., 2008b; Boehmer et al., 2009). The present study describes a method validated using a comparatively larger number of cows (16 in total), collected data for a longer period ( $4 \mathrm{~d}$ in total), and carrying out observations under natural (e.g., not forced to drink) conditions. More importantly, we validated our method across a wide range of values $\left(35.7^{\circ} \mathrm{C}\right.$ to $39.5^{\circ} \mathrm{C}$ for the general-fixed, and $n=1, \ldots, 20$ for the cow-day specific).

A general-fixed threshold algorithm may be useful to monitor drinking behavior at the herd level although possible differences between herds should be explored. For example, a herd that shows a sudden decrease in the number of drinking events may indicate a problem in the water supply, whereas a sudden increase in drinking events may be associated with a substantial change in ambient temperature (Polsky and von Keyserlingk, 2017). A cow-day specific threshold algorithm is better suited to monitoring drinking events at the individual animal level, which could be used for health prediction and improving animal welfare. Siivonen et al. (2011) showed that cows with acute mastitis spent more time eating but less time ruminating and drinking, and that those changes occurred at specific times of the day. These changes in individual behavior pattern could be used for disease prediction. A cow-day specific threshold can provide a more reliable method for constructing a history of the drinking patterns for each individual animal, and hence for intra-individual comparison, because this considers the individual variability and the intensity of a behavior, which is important for identifying animals at greater risk of poor health and welfare (Cortés Fernández de Arcipreste et al., 2018; Vázquez Diosdado et al., 2018). Moreover, computing a cow-day specific threshold is very simple, requires only one day of data, and hence does not add any major computational cost.

The ability to detect drinking events using a thresholding method and by incorporating this information rather that removing it, as in previous studies (CooperPrado et al., 2011; Costa et al., 2016), has the potential to expand temperature monitoring in cows (CooperPrado et al., 2011; Timsit et al., 2011; Costa et al., 2016; Kovács et al., 2017). Drinking behavior in dairy cattle is important for the physical and emotional state of the animals (Baxter, 1983) and for farm economics because it is highly related to milk production (Steiger Burgos et al., 2001; Kramer et al., 2009; Daros et al., 2019). Studies have shown that a decrease in water intake occurs during fever and mastitis episodes (De Mol et al., 2001; Lukas et al., 2008), and an increase in drinking events is associated with an increase in THI
(González Pereyra et al., 2010; Ammer et al., 2016b). By monitoring drinking behavior on its own or in combination with other behaviors, it might be possible to improve current methods for the detection of diseases in cows and heat stress. Additionally, monitoring drinking events during disease episodes can provide information on disease prognosis (Wenge et al., 2014). Therefore, monitoring drinking behavior using a simple threshold algorithm becomes very important because it is computationally low cost and could therefore be done in real time, adding a highly accurate behavior prediction (97.07\% maximum accuracy; Supplemental Table S2; https://doi.org/10.3168/jds.2019-16442) and making it easier to be integrated in farms' monitoring systems.

We investigated the association between reticulorumen temperature drop characteristics and environmental and cow factors using the different threshold values for the detection of drinking events. Results of the mixed model analysis showed that ranges of $37.1^{\circ} \mathrm{C}$ to $38.1^{\circ} \mathrm{C}$ and $n=15$ to $n=10$ detected several reticulorumen temperature drops (approximately 6 drinking events) that were close to previously reported values of 7.3 drinking events per day (Cardot et al., 2008). The values of the amplitude of reticulorumen temperature drop observed $\left(2.30\right.$ to $\left.3.01^{\circ} \mathrm{C}\right)$ were close to previously reported values of $2.2^{\circ} \mathrm{C}( \pm 0.50)$ when drinking hot water $\left(34.3^{\circ} \mathrm{C}\right.$; Bewley et al., 2008b) but significantly different from an amplitude of $8.5^{\circ} \mathrm{C}( \pm 0.50)$ when drinking cold water $\left(5.1^{\circ} \mathrm{C}\right.$; Bewley et al., 2008b). Additionally, the recovery times of 30 min observed in this study were significantly different from reported values of at least $2 \mathrm{~h}$ when cows drank cold water $\left(16^{\circ} \mathrm{C}\right.$ and $7.6^{\circ} \mathrm{C}$; Bewley et al., 2008b; Boehmer et al., 2009). It is important to consider that previously reported recovery times were observed under controlled environments where cows were not allowed to drink water (21 $\mathrm{h}$ in Boehmer et al., 2009; $2 \mathrm{~h}$ in Bewley et al., 2008b) before the experiment. Also, the shorter recovery time observed in the present study may be explained not only by the fact that cows had ad libitum access to water and feed, but also by the water reticulorumen temperature, which may have been colder than ambient temperature.

As days in milk progressed (DIM 2 and DIM 3), cows showed fewer drops but a greater drop amplitude, meaning that cows with higher DIM had fewer drinking bouts but greater water intake. This finding could be related to changes in drinking behavior pattern associated with the stage of lactation, as cows have a higher water intake particularly between 60 and $180 \mathrm{~d}$ after calving due to the greater milk production (Kramer et al., 2009). Regarding parity, cows in parity 2 and 3 showed fewer drops and larger amplitude of drops than cows in parity 1 , meaning that these cows drank water 
less frequently but had larger water intakes than cows in parity 1. Dado and Allen (1994) observed than multiparous cows had higher water intake than primiparous cows, probably associated with milk production levels.

Interestingly, the number of drops was positively associated with ambient temperature at the cow level, meaning that cows appeared to drink more frequently when the ambient temperature was between 16 and $17^{\circ} \mathrm{C}$. In contrast, the amplitude of the drop was negatively associated with ambient temperature, being smaller when ambient temperature was between 16 and $17^{\circ} \mathrm{C}$. Previous studies have shown that water temperature has a positive effect on the amplitude of temperature drop and on recovery time (Bewley et al., 2008b; Boehmer et al., 2009). Other predictor variables such as milk production and hour of the day did not have any significant effect on the different drop characteristics. However, only average monthly milk production data were used in our study, which may not have allowed us to control for daily individual variation in the analysis.

The data to validate our threshold method were based on behavioral data collected by an experienced dairy cattle behavior scientist using the definition of drinking behavior from Cardot et al. (2008); considering that drinking behavior occurs at frequency of approximately 8 events per day, it is easy to detect reliably, even by untrained observers (Martin and Bateson, 2007). Additionally, although the present study was carried out on a single farm, the cow-day specific threshold considers biological variation within cow; therefore, its applicability could be generalized to other similar environments. However, it is important to consider that individual farm ambient and water temperature data, including local seasonality, must be considered part of the method. Particularly, as demonstrated by the present and previous studies, these factors affect directly the drop in temperature and the extent of recovery (Bewley et al., 2008b; Boehmer et al., 2009; Cantor et al., 2018). Further research is needed to understand the effects of water quantity in rumen temperature and type of management system (e.g., restricted access to water) in the accuracy of the threshold method, particularly as a monitoring and predictive tool.

\section{CONCLUSIONS}

We demonstrated in this study how drinking behavior can be detected at a high performance level using a simple thresholding algorithm, and how selection of the threshold value can affect the detection performance. Using a cow-day specific threshold with a threshold of $\mu-\sigma$ (mean cow-day reticuloruminal temperature minus $1 \mathrm{SD}$ ), we obtained the best detection performance and the closest number of predicted drinking events.
The findings of this study provide useful information for the selection of a threshold value of reticuloruminal temperature to detect drinking events and further our understanding of associations between environmental or animal factors and drinking behavior. Future studies should look into integrating reticuloruminal temperature drops as a proxy for drinking behavior to monitor and predict health and physiological status of dairy cattle.

\section{ACKNOWLEDGMENTS}

The authors thank Jurgen Mitsch (Advanced Data Analysis Centre, University of Nottingham, UK). The authors are grateful for the assistance of PrognostiX (Exmouth, United Kingdom) staff. This study was funded by Innovate UK (Swindon, United Kingdom, project 103356) with British Telecommunications and PrognostiX as industrial partners and University of Nottingham as the academic lead.

\section{REFERENCES}

Ammer, S., C. Lambertz, and M. Gauly. 2016a. Comparison of different measuring methods for body temperature in lactating cows under different climatic conditions. J. Dairy Res. 83:165-172.

Ammer, S., C. Lambertz, and M. Gauly. 2016b. Is reticular temperature a useful indicator of heat stress in dairy cattle? J. Dairy Sci. 99:10067-10076.

Bates, D., M. Mächler, B. Bolker, and S. Walker. 2015. Fitting linear mixed-effects models using lme4. 67:48.

Baxter, M. R. 1983. Ethology in environmental design for animal production. Appl. Anim. Ethol. 9:207-220.

Ben-David, A. 2008. Comparison of classification accuracy using Cohen's weighted kappa. Expert Syst. Appl. 34:825-832.

Berckmans, D. 2014. Precision livestock farming technologies for welfare management in intensive livestock systems. Rev. Sci. Tech. 33:189-196.

Bewley, J. M., M. E. Einstein, M. W. Grott, and M. M. Schutz. 2008a. Comparison of reticular and rectal core body temperatures in lactating dairy cows. J. Dairy Sci. 91:4661-4672.

Bewley, J. M., M. W. Grott, M. E. Einstein, and M. M. Schutz. 2008b. Impact of intake water temperatures on reticular temperatures of lactating dairy cows. J. Dairy Sci. 91:3880-3887.

Boehmer, B. H., C. L. Bailey, E. C. Wright, and R. P. Wettemann 2009. Effects of temperature of consumed water on rumen temperature of beef cows. Oklahoma Agricultural Experiment Station, Stillwater.

Box, G. E. P., and D. R. Cox. 1964. An analysis of transformation. J. R. Stat. Soc. B 26:211-252.

Cantor, M. C., J. H. C. Costa, and J. M. Bewley. 2018. Impact of observed and controlled water intake on reticulorumen temperature in lactating dairy cattle. Animals (Basel) 8:E194.

Cardot, V., Y. Le Roux, and S. Jurjanz. 2008. Drinking behavior of lactating dairy cows and prediction of their water intake. J. Dairy Sci. 91:2257-2264.

Cooper-Prado, M. J., N. M. Long, E. C. Wright, C. L. Goad, and R. P. Wettemann. 2011. Relationship of ruminal temperature with parturition and estrus of beef cows. J. Anim. Sci. 89:1020-1027.

Cortés Fernández de Arcipreste, N., K. F. Mancera, G. G. MiguelPacheco, and F. Galindo. 2018. Plasticity and consistency of lying and ruminating behaviours of heifers exposed to different cubicle availability: A glance at individuality. Appl. Anim. Behav. Sci. 205:1-7. 
Costa, J. B. G., J. K. Ahola, Z. D. Weller, R. K. Peel, J. C. Whittier, and J. O. J. Barcellos. 2016. Reticulo-rumen temperature as a predictor of calving time in primiparous and parous Holstein females. J. Dairy Sci. 99:4839-4850.

Dado, R. G., and M. S. Allen. 1994. Variation in and relationships among feeding, chewing, and drinking variables for lactating dairy cows. J. Dairy Sci. 77:132-144.

Daros, R. R., J. A. Bran, M. J. Hotzel, and M. A. G. von Keyserlingk. 2019. Readily available water access is associated with greater milk production in grazing dairy herds. Animals (Basel) 9:E48. https:/ /doi.org/10.3390/ani9020048.

De Mol, R., W. Ouweltjes, G. Kroeze, and M. Hendriks. 2001. Detection of estrus and mastitis: Field performance of a model. Appl. Eng. Agric. 17:399-407.

Dohoo, I. R., W. Martin, and H. Stryhn. 2009. Veterinary Epidemiologic Research. 2nd ed. No. V413 DOHv. AVC Inc. Charlottetown, PEI, Canada.

Gasteiner, J., M. Fallast, S. Rosenkranz, J. Häusler, K. Schneider, and T. Guggenberger. 2009. Measuring rumen $\mathrm{pH}$ and temperature by an indwelling and wireless data transmitting unit and application under different feeding conditions. Pages 127-133 in Proc. Livestock Precision Farming. Wageningen Publishers, Wageningen, the Netherlands.

González Pereyra, A. V., V. Maldonado May, C. G. Catracchia, M. A. Herrero, M. C. Flores, and M. Mazzini. 2010. Influence of water temperature and heat stress on drinking water intake in dairy cows. Chil. J. Agric. Res. 70:328-336.

Koltes, J. E., D. A. Koltes, B. E. Mote, J. Tucker, and I. I. I. D. S. Hubbell. 2018. Automated collection of heat stress data in livestock: New technologies and opportunities. Transl. Anim. Sci. $2: 319-323$.

Kovács, L., F. L. Kézér, F. Ruff, and O. Szenci. 2017. Rumination time and reticuloruminal temperature as possible predictors of dystocia in dairy cows. J. Dairy Sci. 100:1568-1579.

Kramer, E., E. Stamer, J. Spilke, G. Thaller, and J. Krieter. 2009. Analysis of water intake and dry matter intake using different lactation curve models. J. Dairy Sci. 92:4072-4081.

Lees, A. M., J. C. Lees, A. T. Lisle, M. L. Sullivan, and J. B. Gaughan. 2018. Effect of heat stress on rumen temperature of three breeds of cattle. Int. J. Biometeorol. 62:207-215.

Liang, D., C. L. Wood, K. J. McQuerry, D. L. Ray, J. D. Clark, and J. M. Bewley. 2013. Influence of breed, milk production, season, and ambient temperature on dairy cow reticulorumen temperature. J. Dairy Sci. 96:5072-5081.

Lukas, J. M., J. K. Reneau, and J. G. Linn. 2008. Water intake and dry matter intake changes as a feeding management tool and indicator of health and estrus status in dairy cows. J. Dairy Sci. 91:3385-3394

Martin, P., and P. Bateson. 2007. Measuring Behaviour: An Introductory Guide. 3rd ed. Cambridge University Press, Cambridge, UK.

Maruo, K., Y. Yamaguchi, H. Noma, and M. Gosho. 2017. Interpretable inference on the mixed effect model with the Box-Cox transformation. Stat. Med. 36:2420-2434.
Matlab and Statistics Toolbox Release. 2017a. The MathWorks Inc. Natick, MA.

Murphy, M. R. 1992. Water metabolism of dairy cattle. J. Dairy Sci. 75:326-333.

Polsky, L., and M. A. G. von Keyserlingk. 2017. Invited review: Effects of heat stress on dairy cattle welfare. J. Dairy Sci. 100:8645-8657.

R Core Team. 2017. R: A language and environment for statistical computing. R Foundation for Statistical Computing, Vienna, Austria.

Sakia, R. M. 1992. The Box-Cox transformation technique: A review. Statistician 41:169-178. https://doi.org/10.2307/2348250.

Seaks, T. G., and S. Layson. 1983. Box Cox Estimation with Standard Econometric Problems. Vol. 65.

Siivonen, J., S. Taponen, M. Hovinen, M. Pastell, B. J. Lensink, S. Pyörälä, and L. Hänninen. 2011. Impact of acute clinical mastitis on cow behaviour. Appl. Anim. Behav. Sci. 132:101-106.

SmaXtec. 2018. Smaxtec basic bolus. Accessed July 1, 2018. https:// www.smaxtec.com/en/smaxtec-basic-bolus/.

Steiger Burgos, M., M. Senn, F. Sutter, M. Kreuzer, and W. Langhans. 2001. Effect of water restriction on feeding and metabolism in dairy cows. Am. J. Physiol. Regul. Integr. Comp. Physiol 280:R418-R427.

Stone, A. E., B. W. Jones, C. A. Becker, and J. M. Bewley. 2017. Influence of breed, milk yield, and temperature-humidity index on dairy cow lying time, neck activity, reticulorumen temperature, and rumination behavior. J. Dairy Sci. 100:2395-2403.

Timsit, E., S. Assie, R. Quiniou, H. Seegers, and N. Bareille. 2011 Early detection of bovine respiratory disease in young bulls using reticulo-rumen temperature boluses. Vet. J. 190:136-142.

Tong, H. 2011. Threshold models in time series analysis - 30 years on. Stat. Interface 4:107-118.

Vázquez Diosdado, J. A., Z. E. Barker, H. R. Hodges, J. R. Amory, D. P. Croft, N. J. Bell, and E. A. Codling. 2015. Classification of behaviour in housed dairy cows using an accelerometer-based activity monitoring system. Anim. Biotelem. 3:15

Vázquez Diosdado, J. A., Z. E. Barker, H. R. Hodges, J. R. Amory, D. P. Croft, N. J. Bell, and E. A. Codling. 2018. Space-use patterns highlight behavioural differences linked to lameness, parity, and days in milk in barn-housed dairy cows. PLoS One 13:e208424.

Venables, W. N., and B. D. Ripley. 2002. Modern Applied Statistics with S. Fourth ed. Springer, New York.

Walton, E., C. Casey, J. Mitsch, J. A. Vázquez-Diosdado, J. Yan, T. Dottorini, K. A. Ellis, A. Winterlich, and J. Kaler. 2018. Evaluation of sampling frequency, window size and sensor position for classification of sheep behaviour. R. Soc. Open Sci. 5:171442.

Wenge, J., I. Steinhöfel, C. Heinrich, M. Coenen, and L. Bachmann. 2014. Water and concentrate intake, weight gain and duration of diarrhea in young suckling calves on different diets. Livest. Sci. 159:133-140. 